Artigo Original

Original Article

\section{Avaliação da atenção primária em saúde por usuários com e sem deficiência}

\author{
Primary health care assessment by users with \\ and without disabilities
}

Descritores

Atenção Primária à Saúde

Pessoas com Deficiência

Acesso aos Serviços de Saúde

Integralidade em Saúde

Pesquisa sobre Serviços de Saúde

Sistema Único de Saúde

Keywords

Primary Health Care Disabled Individuals Health Services Accessibility Integrality in Health Health Services Research Unified Health System

\section{Endereço para correspondência:} Daniela Regina Molini-Avejonas Universidade de São Paulo - USP Rua Cipotânea, 51, São Paulo (SP), Brasil, CEP: 05360-160. E-mail: danielamolini@usp.br

Recebido em: Maio 17, 2017

Aceito em: Novembro 09, 2017

\section{RESUMO}

Objetivo: Avaliar atributos essenciais (acesso de primeiro contato, longitudinalidade, integralidade e coordenação da atenção) e derivados (orientação familiar e comunitária) da Atenção Primária em Saúde (APS) na percepção de usuários com e sem deficiência. Método: Estudo transversal observacional utilizando o questionário Primary Care Assesment Tool em usuários autodeclarados com e sem deficiência de cinco unidades básicas de saúde de um município no qual 55\% da população depende do SUS. Foram atribuídos escores para as respostas dadas a cada atributo de atenção primária à saúde avaliado. Resultados: Participaram 93 usuários (67 com e 26 sem deficiência). Não houve diferença estatisticamente significante para nenhum dos atributos na comparação entre pessoas com e sem deficiência. Para o grupo como um todo, obtiveram-se escores acima do critério de corte: Grau de afiliação, Acesso de primeiro contato (utilização), Longitudinalidade e Coordenação da atenção (sistema de informação). Receberam pior avaliação: Acesso de primeiro contato (acessibilidade), Coordenação da atenção (integralidade do cuidado), Integralidade (serviços disponíveis e prestados) e Orientação familiar e comunitária. Os usuários reconhecem que as equipes identificam problemas de locomoção e movimentação, mas que existem falhas no reconhecimento de problemas para ouvir, falar e enxergar; e na orientação das pessoas que necessitam de auxílio e das que prestam cuidados. Conclusão: Os usuários com e sem deficiência avaliaram de forma semelhante a atenção que recebem e indicaram fragilidades no reconhecimento de demandas específicas. Há necessidade de mudanças (estruturais e do processo de trabalho) para assegurar acessibilidade, integralidade do cuidado e orientação familiar e comunitária e, assim, melhor qualificar a APS.

\begin{abstract}
Purpose: To evaluate the core (First Contact, Longitudinality, Comprehensiveness, and Coordination of Services) and derivative (Family Orientation, Community Orientation) attributes of primary health care (PHC) from the perspective of users with and without disabilities. Methods: Observational, cross-sectional study using the Primary Care Assessment Tool (PCAT) with users with and without disabilities of five basic health units (BHU) in a municipality where $55 \%$ of the population depends on the Brazilian Unified Health System (SUS). Scores were assigned to the responses given to each of the PHC attributes evaluated. Results: Study participants were 93 (67 physically disabled and 26 without disabilities) PHC users. No statistically significant differences were observed for any attribute on the comparison between the populations. For both groups, the attributes Degree of Affiliation, First Contact - Utilization, Longitudinality, and Coordination of Services - Information System received satisfactory (above cutoff) scores, whereas the attributes First Contact - Accessibility, Coordination of Services - Care Integration, Comprehensiveness, Family Orientation, and Community Orientation received unsatisfactory (below cutoff) scores. Users reported that the health teams are able to satisfactorily identify mobility issues, but there are failures in the recognition of problems of hearing, voice/speech and vision, and in the orientation of services available and services provided. Conclusion: Users with and without disabilities evaluated the health care received similarly, indicating fragilities on the recognition of specific demands. Structural and work process changes should be conducted to ensure Accessibility, Comprehensiveness, and Family and Community Orientation, and thus increase the quality of PHC.
\end{abstract}

Trabalho realizado no Departamento de Fisioterapia, Fonoaudiologia e Terapia Ocupacional da Faculdade de Medicina, Universidade de São Paulo - USP - São Paulo (SP), Brasil.

${ }^{1}$ Universidade de São Paulo - USP - São Paulo (SP) Brasil.

Fonte de financiamento: Edital PPSUS 2013 FAPESP 2014/50012-8 e Programa de Educação pelo Trabalho na Saúde - Redes de Atenção (PET/Redes 2013-2015).

Conflito de interesses: nada a declarar. 


\section{INTRODUÇÃO}

A Atenção Primária em Saúde (APS) é parte integrante e estratégica do sistema de saúde. No Brasil, o desenvolvimento de serviços desse nível de atenção foi orientado por diferentes modelos; dentre eles destacam-se Ações Programáticas em Saúde ${ }^{(1,2)}$ e, mais recentemente, a Estratégia de Saúde da Família $(\mathrm{ESF})^{(3)}$, com o objetivo de reformulação do modelo assistencial para efetivação do Sistema Único de Saúde (SUS).

$\mathrm{Na}$ Política Nacional de Atenção Básica ${ }^{(3)}$, estão indicados os princípios essenciais para o desenvolvimento da APS na perspectiva desafiadora da universalização do acesso e da atenção à saúde da população com equidade, integralidade e participação dos usuários, necessárias para a garantia do direito à saúde.

A expansão da APS tem ainda como desafio o enfrentamento das mudanças ocorridas no perfil demográfico e de morbimortalidade da população brasileira. Em resposta ao aumento da prevalência das doenças crônicas não transmissíveis e que incluem os vários tipos de deficiências, espera-se que a APS atue como coordenadora do cuidado e ordenadora da rede de serviços ${ }^{(4)}$.

Nesse sentido, há necessidade de avaliação do desenvolvimento da APS para responder a estas demandas específicas. Uma das iniciativas voltadas para esse fim refere-se à utilização do Primary Care Assesment Tool (PCATool), instrumento validado no país e indicado, desde 2010, pelo Ministério da Saúde como uma das estratégias para avaliação e aprimoramento dos serviços de atenção básica em saúde ${ }^{(5)}$.

O PCATool, instrumento desenvolvido por Starfield ${ }^{(6)}$, é voltado para a avaliação dos atributos essenciais e derivados da atenção primária em saúde e vem sendo utilizado em muitos países, demonstrando ser sensível para os objetivos a que se propõe. Existem, na literatura internacional e nacional, outros instrumentos para avaliação de atenção primária, entretanto, os autores de um artigo que compila os questionários disponíveis concluem que o PCATool é o mais adequado para esta avaliação, pois permite avaliar a atenção à saúde centrada na família e na comunidade $^{(7)}$ o que corrobora a Política Nacional de Atenção Básica Brasileira ${ }^{(3)}$.

Nesse processo de avaliação, é fundamental conhecer a percepção dos usuários quanto à oferta e qualidade dos serviços prestados, o que possibilita seu aperfeiçoamento e reorientação. Desta forma, diversos estudos exploraram esta questão, sob a perspectiva dos usuários adultos, responsáveis por crianças ou idosos. Outros, ainda, estabeleceram relações entre percepções de distintas populações (1,2,8-17) $^{(2 .}$

No entanto, identifica-se um único estudo no Brasil que avalia a percepção de pessoas com deficiência sobre diferentes atributos da atenção primária em saúde ${ }^{(18)}$. Assim, devido à escassez de estudos sobre pessoas com deficiência, ao histórico de desenvolvimento de serviços especializados para sua atenção, estreitamente vinculados às iniciativas filantrópicas e beneficentes, e à expectativa e necessidade de que serviços de atenção primária identifiquem e acompanhem essas populações em suas diferentes necessidades de saúde, evidencia-se a importância de estudos relacionados aos usuários com deficiências na APS. Por esses motivos, são objetivos deste estudo: a) avaliar os atributos essenciais e derivados da atenção básica, a partir da percepção de usuários, em especial daqueles com deficiência, de unidades básicas de saúde na região oeste do município de São Paulo e que possuem parceria com a Universidade de São Paulo em projetos de articulação ensino-serviço, b) conhecer alguns fatores relacionados à percepção dos usuários em relação aos atributos da atenção básica.

\section{MÉTODO}

Trata-se de estudo transversal e observacional, desenvolvido entre agosto e dezembro de 2014. Este estudo, parte integrante da pesquisa "Atenção Básica como ordenadora das redes de atenção à saúde Cegonha e de Cuidados à Pessoa com Deficiência", teve aprovação dos Comitês de Ética da Secretaria Municipal de Saúde de São Paulo (32273014.8.0000.0065) e da Faculdade de Medicina da Universidade de São Paulo (processo no 189/14), contou com apoio da FAPESP, por meio do Edital PPSUS 2014/50012-8, e também do Ministério da Saúde (MS), através de projeto de integração ensino-serviço: Programa de Educação pelo Trabalho na Saúde - PET Saúde/Redes de Atenção. Esse Programa compreendeu parceria entre Secretaria Municipal de Saúde de São Paulo e Universidade de São Paulo - (2013-2015) para o acompanhamento, por estudantes, de atividades assistenciais próprias do cotidiano dos serviços e desenvolvimento de pesquisa voltada a avaliação e fortalecimento de redes de atenção à saúde. O Programa contou com a concessão pelo MS de bolsas para estudantes, preceptores dos serviços e tutores da Universidade.

\section{Cenário da pesquisa}

O estudo foi desenvolvido em unidades básicas de saúde (UBS) situadas na área da Supervisão Técnica de Saúde do Butantã da Secretaria Municipal de Saúde de São Paulo. A supervisão técnica de saúde da região tinha sob sua responsabilidade uma população de cerca de 428.217 habitantes e, na Atenção Básica, contava com 14 unidades básicas de saúde. Cinco dessas unidades foram selecionadas para o estudo pela parceria com a Universidade de São Paulo em projetos de articulação ensino-serviço, particularmente por sua vinculação ao Programa PET - Saúde/Redes de Atenção. Essa parceria não implica provisão de recursos humanos e materiais por parte da Universidade, porém é essencial para a formação em saúde por oferecer cenários de práticas para estudantes participarem de atividades previstas para APS pela Secretaria Municipal da Saúde. Essas UBS possuíam características diferentes no que diz respeito à atenção que realizavam às pessoas com deficiência e seus familiares. Duas UBS funcionam exclusivamente com a Estratégia de Saúde da Família (ESF) e três são mistas, por atenderem nas modalidades ESF e Modelo Tradicional de assistência. Além das atribuições no âmbito da ESF, as UBS também realizam outras atividades assistenciais que incluem práticas corporais e outras abordagens complementares. Quatro unidades contam com o Núcleo de Apoio à Saúde da Família (NASF) e uma possui equipe multidisciplinar específica. O desenvolvimento de atividades a partir da ESF e o apoio por equipe multidisciplinar foram critérios de inclusão das unidades no estudo. 


\section{Casuística}

A população integrante do estudo foi indicada como sendo de pessoas com deficiência pelos profissionais das equipes multiprofissionais dos serviços, vinculados ao Programa PET - Saúde/Redes de Atenção. Durante a aplicação dos questionários, uma pequena parte dessa população não declarou possuir algum tipo de deficiência, sendo, desta forma, identificada como "sem deficiência". Os demais se autodeclaram como pessoas com deficiência. Desta forma, os indivíduos do estudo foram divididos em dois grupos: G1 (constituído por pessoas autodeclaradas com deficiência) e G2 (pessoas que não se declararam com deficiência), de distintas faixas etárias, moradores da área de abrangência das cinco UBS participantes, acompanhados pelas equipes de saúde dessas unidades. Conforme previsto pelo Instrumento de pesquisa, os adultos responsáveis por menores de 18 anos, assim como os responsáveis por pessoas com deficiência mental severa, responderam aos questionários. Todos assinaram o Termo de Consentimento Livre e Esclarecido antes da aplicação do questionário.

\section{Procedimentos}

Os dados foram coletados por meio de entrevistas com a aplicação do PCATool. Esse instrumento avalia os seguintes atributos essenciais da APS: Acesso de primeiro contato, que implica acessibilidade e uso de serviços para cada novo problema ou episódio; Longitudinalidade, que pressupõe a existência de uma fonte regular de cuidados e seu uso ao longo do tempo; Integralidade, significando um leque de serviços disponíveis e prestados pelo serviço de atenção primária, voltados à atenção integral (incluindo encaminhamentos a serviços secundários, terciários e de apoio); e Coordenação de cuidados, que requer alguma forma de continuidade, reconhecimento de problemas tratados em outros serviços e a integração destes cuidados no acolhimento total do paciente. Os atributos derivados identificados e avaliados pelo PCATool qualificam as ações dos serviços, sendo eles: orientação familiar, orientação comunitária e competência cultural ou adaptação dos profissionais de saúde às características culturais da população( ${ }^{(6)}$.

O PCATool prevê cinco respostas possíveis para cada item que compõe o atributo ou suas dimensões: "com certeza sim" $($ valor $=4)$, "provavelmente sim" (valor $=3$ ), "provavelmente não" (valor = 2), "com certeza não" (valor =1) e "não sei/não lembro" (valor $=9$ ). O valor obtido em cada item é transformado em escala de 0 a 10, por meio do seguinte cálculo: [(escore obtido -1$) \times 10] / 3$. Valores de escores $\geq 6,6$ são considerados elevados e equivalentes ao valor $\geq 3$ na escala Likert ${ }^{(19)}$.

O escore de cada atributo, ou de suas dimensões, é calculado a partir da média dos valores das respostas dos itens. Há alguns itens que, quanto maior o escore atribuído, menor é a sua orientação para APS e vice-versa. Nestes casos, os itens devem ter seus valores invertidos. Quando um entrevistado atinge 50\% ou mais do total de itens com respostas em branco, o escore deste entrevistado não deve ser calculado. Se a soma das respostas em branco for menor que $50 \%$, estas devem ser transformadas de valor 9 ("não sei/não lembro") para valor 2 ("provavelmente não").

No caso do atributo Grau de Afiliação, o escore possui uma escala própria, com valores de 1 a 4 não transformáveis numa escala de 1 a 10, em que o 1 representa afiliação a quatro serviços diferentes e 4, afiliação a um único serviço em todas as questões.

O Escore Essencial de APS é medido pela soma do escore médio das dimensões que pertencem aos atributos essenciais mais o grau de afiliação dividido pelo número de dimensões. O Escore Geral de APS é medido pela soma do escore médio das dimensões que pertencem aos atributos essenciais mais as dimensões que pertencem aos atributos derivados mais o grau de afiliação dividido pelo número total de dimensões ${ }^{(19)}$.

O PCATool foi complementado por um roteiro de perguntas voltadas à caracterização demográfica dos usuários, presença e tipos de deficiência, reconhecimento e acompanhamento dessas no serviço por profissionais de equipe multidisciplinar (exceto médicos e enfermeiros), pela Classificação socioeconômica das famílias e grau de instrução de seu chefe da Associação Brasileira de Empresas de Pesquisa ${ }^{(20)}$, inserção na escola, trabalho e/ou em grupos comunitários.

$\mathrm{O}$ instrumental foi aplicado durante três semanas típicas, por 45 minutos a 1 hora por estudantes bolsistas do Programa PET Saúde/Redes, treinados pelos pesquisadores e acompanhados por preceptores/profissionais dos serviços.

\section{ANÁLISE DOS DADOS}

Os dados numéricos foram expressos por meio de médias e desvio padrão. O pressuposto da distribuição normal de cada variável foi avaliado com o teste de Shapiro-Wilk. Considerando a distribuição não normal, recorreu-se ao teste Mann Whitney, para realização das comparações entre dois grupos, e ao teste Kruskal Wallis, para as comparações entre três ou mais grupos. Foi considerada uma probabilidade de erro do tipo I $(\alpha)$ de 0,05 em todas as análises inferenciais. Para as análises, foi utilizado o software SPSS versão 21 (SPSS 21.0 for Windows).

\section{RESULTADOS}

A caracterização sociodemográfica dos 93 usuários encontra-se na Tabela 1.

A amostra foi composta por 67 usuários (72,04\%) autodeclarados com alguma deficiência e 26 (27,95\%) autodeclarados sem deficiência. Dentre os usuários com deficiência, 29,33\% têm deficiência intelectual; 24\%, deficiência física; 13,33\%, deficiência motora; $12 \%$, deficiências múltiplas; 5,33\%, deficiência auditiva; $5,33 \%$, deficiência física associada à intelectual; 4\%, deficiência física associada a alterações da comunicação; $2,67 \%$, deficiência visual; $1,34 \%$, deficiência auditiva e visual; e 2,67\% têm outras deficiências.

A maioria dos usuários são mulheres, com mais de 18 anos (idade mínima de 2 anos e máxima de 80 anos) e solteiros. A maioria dos chefes de família tem o ensino médio completo, bem como a maioria dos adultos e idosos que trabalham possui carteira assinada. Dentre os que não trabalham, a situação mais presente é de aposentadoria. Dentre os usuários menores de 18 anos, mais de $25 \%$ não frequentam a escola e, dos que frequentam, a maioria está em escola pública. Em relação à participação em grupos comunitários, quase a metade apresenta esta vinculação (Tabela 1).

Quanto à classificação socioeconômica, 68,82\% pertencem à classe $\mathrm{C} ; 22,59 \%$, à classe $\mathrm{B} ; 5,37 \%$, à classe $\mathrm{D} ; 2,15 \%$, à classe $\mathrm{A}$; e 1,07\%, à classe $\mathrm{E}$. 
Tabela 1. Caracterização dos usuários, segundo presença de deficiência, gênero, faixa etária, grau de instrução, situação de trabalho, estado civil e participação social

\begin{tabular}{|c|c|c|c|c|}
\hline & Com Deficiência $(n=67)$ & Sem Deficiência $(n=26)$ & Total & Porcentagem \\
\hline \multicolumn{5}{|c|}{ IDADE $(n=93)$} \\
\hline$<18$ anos & 10 & 5 & 15 & 16,12 \\
\hline 18 a 59 anos & 37 & 15 & 52 & 55,92 \\
\hline$>60$ anos & 20 & 6 & 26 & 27,96 \\
\hline \multicolumn{5}{|c|}{ GÊNERO $(n=93)$} \\
\hline Feminino & 41 & 16 & 57 & 61,29 \\
\hline Masculino & 26 & 10 & 36 & 38,71 \\
\hline \multicolumn{5}{|c|}{ ESTADO CIVIL DE ADULTOS E IDOSOS $(n=78)$} \\
\hline Solteiro & 21 & 10 & 31 & 39,74 \\
\hline Casado & 18 & 6 & 24 & 30,77 \\
\hline União Estável & 1 & 0 & 1 & 1,28 \\
\hline Divorciado & 3 & 2 & 5 & 6,41 \\
\hline Viúvo & 7 & 2 & 9 & 11,54 \\
\hline Sem informação & 6 & 2 & 8 & 10,26 \\
\hline \multicolumn{5}{|c|}{ GRAU DE INSTRUÇÃO DO CHEFE DE FAMILIA $(n=93)$} \\
\hline Analfabeto ou até $3 a$ série & 11 & 1 & 12 & 12,90 \\
\hline Fundamental Incompleto & 12 & 8 & 20 & 21,51 \\
\hline Fundamental completo & 18 & 8 & 26 & 27,95 \\
\hline Ensino Médio completo & 21 & 9 & 30 & 32,26 \\
\hline Superior Completo & 4 & 0 & 4 & 4,30 \\
\hline Sem dados & - & - & 1 & 1,08 \\
\hline \multicolumn{5}{|c|}{ SITUAÇÃO DE TRABALHO DE ADULTOS E IDOSOS $(n=33)$} \\
\hline Autônomo & 7 & 1 & 8 & 24,24 \\
\hline Carteira Assinada & 9 & 10 & 19 & 57,58 \\
\hline Informal & 2 & 2 & 4 & 12,12 \\
\hline Outros & 1 & 1 & 2 & 6,06 \\
\hline \multicolumn{5}{|c|}{ SITUAÇÃO DOS ADULTOS E IDOSOS QUE NÃO REALIZAM TRABALHO REMUNERADO $(n=45)$} \\
\hline Auxílio doença & 0 & 1 & 1 & 2,22 \\
\hline Aposentado & 16 & 2 & 18 & 40,00 \\
\hline Benefício de Prestação Continuada & 3 & 0 & 3 & 6,67 \\
\hline Desempregado & 6 & 2 & 8 & 17,78 \\
\hline Cuidados com a casa/Família & 9 & 1 & 10 & 22,22 \\
\hline Outros & 2 & 1 & 3 & 6,67 \\
\hline Não respondeu & 0 & 2 & 2 & 4,44 \\
\hline \multicolumn{5}{|c|}{ PARTICIPAÇÃO DE ADULTOS E IDOSOS EM GRUPOS COMUNITÁRIOS $(n=78)$} \\
\hline Grupo religioso & 7 & 1 & 8 & 10,26 \\
\hline Cooperativa & 0 & 2 & 2 & 2,56 \\
\hline Associações & 1 & 1 & 2 & 2,56 \\
\hline Em mais de um grupo & 2 & 0 & 2 & 2,56 \\
\hline Outros & 18 & 3 & 21 & 26,92 \\
\hline Não frequenta grupos & 8 & 5 & 13 & 16,70 \\
\hline Sem informação & 20 & 10 & 30 & 38,46 \\
\hline \multicolumn{5}{|c|}{ VINCULAÇÃO ESCOLAR DAS CRIANÇAS $(n=15)$} \\
\hline Frequenta escola pública & 5 & 2 & 7 & 46,67 \\
\hline Frequenta escola privada & 1 & 0 & 1 & 6,66 \\
\hline Frequenta escola não especificada & 1 & 0 & 1 & 6,66 \\
\hline Não frequenta escola & 2 & 2 & 4 & 26,67 \\
\hline Sem informação & 1 & 0 & 1 & 13,34 \\
\hline
\end{tabular}


Tabela 2. Médias dos escores (e DP) dos atributos e dimensões referentes à pontuação obtida pelo PCATool dos usuários sem e com deficiência; p-valor

\begin{tabular}{|c|c|c|c|c|}
\hline Atributo & Dimensão & $\begin{array}{c}\text { Usuários sem deficiência } \\
\text { Média } \\
\text { (DP) } \\
\end{array}$ & $\begin{array}{c}\text { Usuários com deficiência } \\
\text { Média } \\
\text { (DP) }\end{array}$ & $\mathrm{p}$-valor \\
\hline Grau de Afiliação & --- & $\begin{array}{c}3,13 \\
(1,12)\end{array}$ & $\begin{array}{c}3,30 \\
(1,02)\end{array}$ & 0,953 \\
\hline \multirow{2}{*}{$\begin{array}{l}\text { Acesso de primeiro } \\
\text { contato }\end{array}$} & Utilização & $\begin{array}{l}7,37 \\
(2,83)\end{array}$ & $\begin{array}{c}8 \\
(2,56)\end{array}$ & 0,463 \\
\hline & Acessibilidade & $\begin{array}{c}3,35 \\
(1,50)\end{array}$ & $\begin{array}{c}3,67 \\
(1,53)\end{array}$ & 0,745 \\
\hline \multirow{2}{*}{ Coordenação da atenção } & $\begin{array}{c}\text { Coordenação - } \\
\text { Integralidade de Cuidado }\end{array}$ & $\begin{array}{c}7,65 \\
(2,48)\end{array}$ & $\begin{array}{c}6,82 \\
(2,76)\end{array}$ & 0,334 \\
\hline & $\begin{array}{l}\text { Coordenação - Sistema } \\
\text { de Informação }\end{array}$ & $\begin{array}{c}6,72 \\
(2,66)\end{array}$ & $\begin{array}{c}6,47 \\
(2,01)\end{array}$ & 0,773 \\
\hline Orientação familiar & & $\begin{array}{c}6,71 \\
(3,26)\end{array}$ & $\begin{array}{l}5,85 \\
(3,24)\end{array}$ & 0,451 \\
\hline Orientação Comunitária & & $\begin{array}{l}5,87 \\
(2,70)\end{array}$ & $\begin{array}{l}5,47 \\
(2,57)\end{array}$ & 0,717 \\
\hline Escore Geral & & $\begin{array}{c}6,24 \\
(1,75)\end{array}$ & $\begin{array}{c}6,39 \\
(1,66)\end{array}$ & 0,622 \\
\hline Escore Essencial & & $\begin{array}{c}6,46 \\
(1,69)\end{array}$ & $\begin{array}{c}6,32 \\
(1,52)\end{array}$ & 0,644 \\
\hline
\end{tabular}

Teste Mann Whitney, com nível de significância de 0,05

Legenda: PCATool = Primary Care Assesment Tool; DP = Desvio padrão

\section{A avaliação da Atenção Básica pelos usuários}

A Tabela 2 apresenta as médias dos escores obtidos por meio do PCATool para os usuários com e sem deficiência. Observou-se que não houve diferença estatisticamente significante na comparação entre os escores desses usuários em todos os atributos, dimensões e escores geral e essencial.

Quando os escores foram analisados de forma qualitativa (Tabela 2), identificou-se que os atributos bem avaliados, por ambos os grupos, foram: Grau de Afiliação, Acesso de primeiro contato - utilização, Longitudinalidade e Coordenação da atenção - integralidade. Quanto aos escores geral e essencial, ambos os grupos não avaliaram a atenção primária positivamente.

Como não houve diferença estatisticamente significante para a comparação entre os grupos com e sem deficiência, conforme demonstrado anteriormente, foi realizada uma comparação entre os escores obtidos por faixa etária, incluindo os indivíduos com e sem deficiência. Assim, na Tabela 3, podem-se visualizar as médias das avaliações dos usuários por faixa etária. Observa-se que houve diferença estatisticamente significante apenas para o atributo Acessibilidade, sendo que este foi melhor avaliado pelos responsáveis por menores de 18 anos.

Numa avaliação qualitativa (Tabela 3), observou-se que os atributos Grau de Afiliação; Acesso de primeiro contato - utilização; Longitudinalidade; e Coordenação da atenção -sistema de informação foram bem avaliados, assim como o escore essencial.
No que diz respeito a alguns atributos essenciais e derivados avaliados como insatisfatórios pelos usuários, a partir do critério de corte, encontram-se nas Tabelas 4 e 5 os escores obtidos para cada um deles.

Na Tabela 4 (avaliação por adultos e idosos), verificou-se que os piores escores foram dados para a dimensão Acessibilidade, com itens que receberam pontuações muito baixas: não abrir no fim de semana e à noite, não fornecer possibilidade de contato telefônico ou acesso a algum profissional, nesses períodos, quando o usuário está doente.

No que se refere a integralidade: serviços disponiveis (Tabela 4), entre os itens que contribuíram para o baixo valor da dimensão, destacam-se alguns não disponíveis: colocação de tala e identificação de deficiências visuais e auditivas. Outros itens mal avaliados incluíram: falta de aconselhamento/ tratamento quanto ao uso prejudicial de drogas, ao processo de envelhecimento e de acompanhamento de familiares com incapacidades.

Quanto à integralidade: serviços prestados (Tabela 4), o menor escore esteve relacionado à falta de aconselhamento sobre porte de armas de fogo, segurança infantil no carro e acidentes domésticos como queimaduras, ingestão de substâncias e quedas.

Já no que se refere a responsáveis por menores de 18 anos (Tabela 5), houve menos itens que se apresentaram abaixo do critério de corte, quando comparados com a avaliação feita por adultos e idosos. O pior item avaliado pelos responsáveis 
Tabela 3. Médias dos escores (e DP) dos atributos e dimensões referentes à pontuação obtida pelo PCATool dos usuários divididos por faixa etária; $\mathrm{p}$-valor

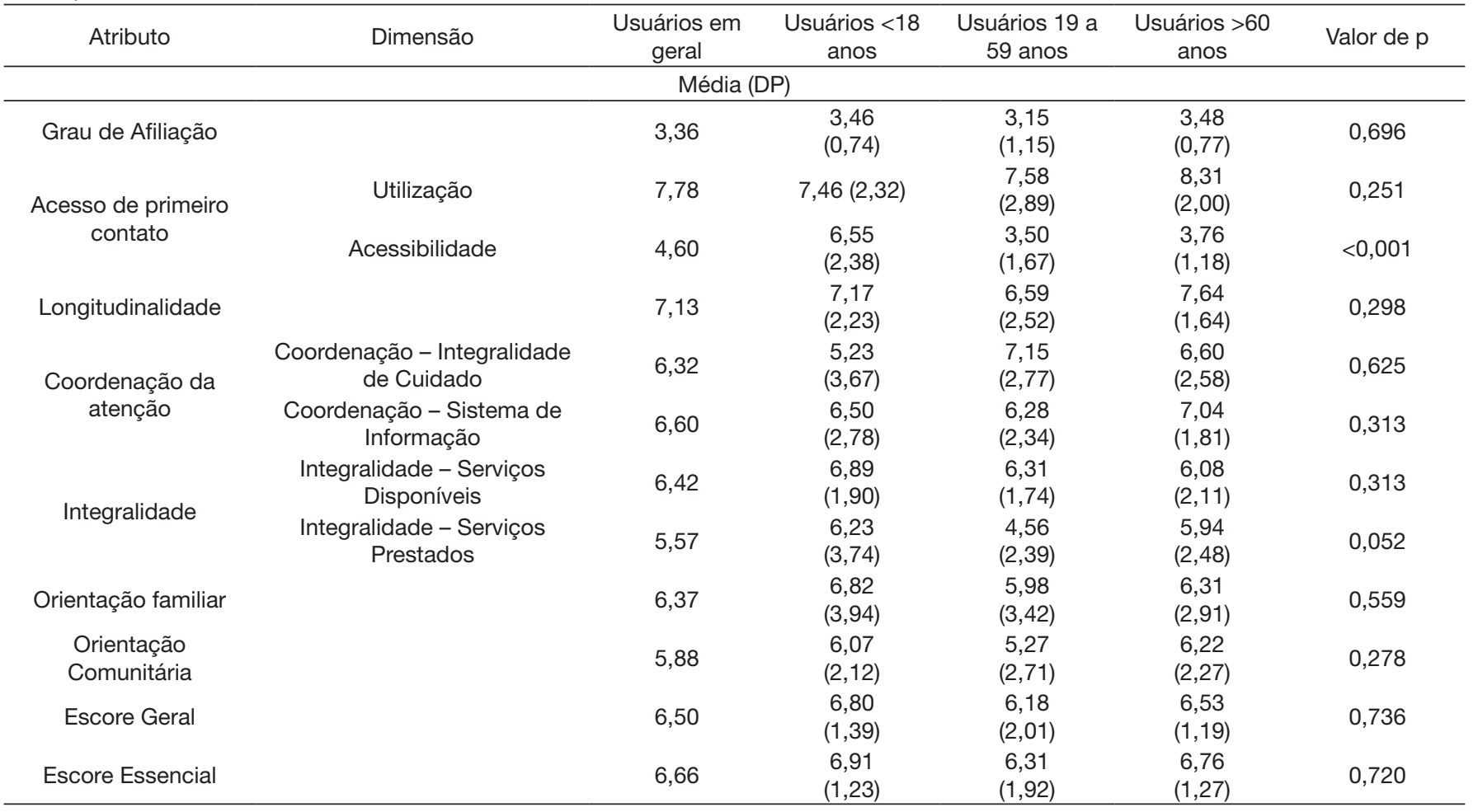

Teste Kruskal Wallis, com nível de significância de 0,05

Legenda: PCATool = Primary Care Assesment Tool; DP = Desvio padrão

Tabela 4. Descrição dos itens que foram avaliados negativamente (abaixo do critério de corte) e que são prioritários para ações de melhoria para adultos e idosos

\begin{tabular}{|c|c|c|c|}
\hline ATRIBUTO & DIMENSÃO & PERGUNTAS & $\begin{array}{l}\text { Escore } \\
\text { médio }\end{array}$ \\
\hline \multirow{8}{*}{$\begin{array}{c}\text { ACESSO DE } \\
\text { PRIMEIRO } \\
\text { CONTATO }\end{array}$} & \multirow{8}{*}{ ACESSIBILIDADE } & C1- O seu serviço de referência fica aberto no sábado ou no domingo? & 0,61 \\
\hline & & $\begin{array}{l}\text { C2 - O seu serviço de referência fica aberto pelo menos algumas noites de dias úteis até as } \\
20 \mathrm{~h} ?\end{array}$ & 1,71 \\
\hline & & $\begin{array}{l}\text { C4 - Quando o seu serviço de referência está aberto, você consegue aconselhamento rápido } \\
\text { pelo telefone se precisar? }\end{array}$ & 2,9 \\
\hline & & $\begin{array}{l}\text { C5 - Quando o seu serviço de referência está fechado, existe um número de telefone para o } \\
\text { qual você possa ligar quando fica doente? }\end{array}$ & 1,17 \\
\hline & & $\begin{array}{l}\text { C7 - Quando o seu serviço de referência está fechado e você fica doente durante a noite, } \\
\text { alguém deste serviço atende você naquela noite? }\end{array}$ & 0,74 \\
\hline & & C8 - É fácil marcar hora para uma consulta de revisão no seu serviço de referência? & 6,36 \\
\hline & & $\begin{array}{l}\text { C9 - Quando você chega no seu serviço de referência, você tem que esperar mais de } 30 \\
\text { minutos para consultar com o médico ou enfermeiro (sem contar atendimento e triagem) }\end{array}$ & 4,07 \\
\hline & & $\begin{array}{l}\text { C12 - Quando você tem que ir ao seu serviço de referência, você tem que faltar ao trabalho ou } \\
\text { à escola para ir ao serviço de saúde? }\end{array}$ & 6,15 \\
\hline \multirow{4}{*}{ COORDENAÇÃO } & \multirow{4}{*}{$\begin{array}{l}\text { INTEGRALIDADE } \\
\text { DO CUIDADO }\end{array}$} & $\begin{array}{l}\text { E4 - O seu "médico/enfermeiro" discutiu com você diferentes serviços onde você poderia ser } \\
\text { atendido para este problema de saúde? }\end{array}$ & 5,56 \\
\hline & & $\begin{array}{l}\text { E5 - O seu "médico / enfermeiro" ou alguém que trabalha no / com "nome do serviço de } \\
\text { saúde" ajudou-o /a a marcar esta consulta? }\end{array}$ & 6,51 \\
\hline & & $\begin{array}{l}\text { E6 - O seu "médico/enfermeiro" escreveu alguma informação para o especialista, a respeito } \\
\text { do motivo desta consulta? }\end{array}$ & 6,59 \\
\hline & & $\begin{array}{l}\text { E9 - O seu "médico/enfermeiro" pareceu interessado na qualidade do cuidado que lhe } \\
\text { foi dado (Ihe perguntou se você foi bem ou mal atendido por este especialista ou serviço } \\
\text { especializado)? }\end{array}$ & 6,11 \\
\hline
\end{tabular}


Tabela 4. Continuação...

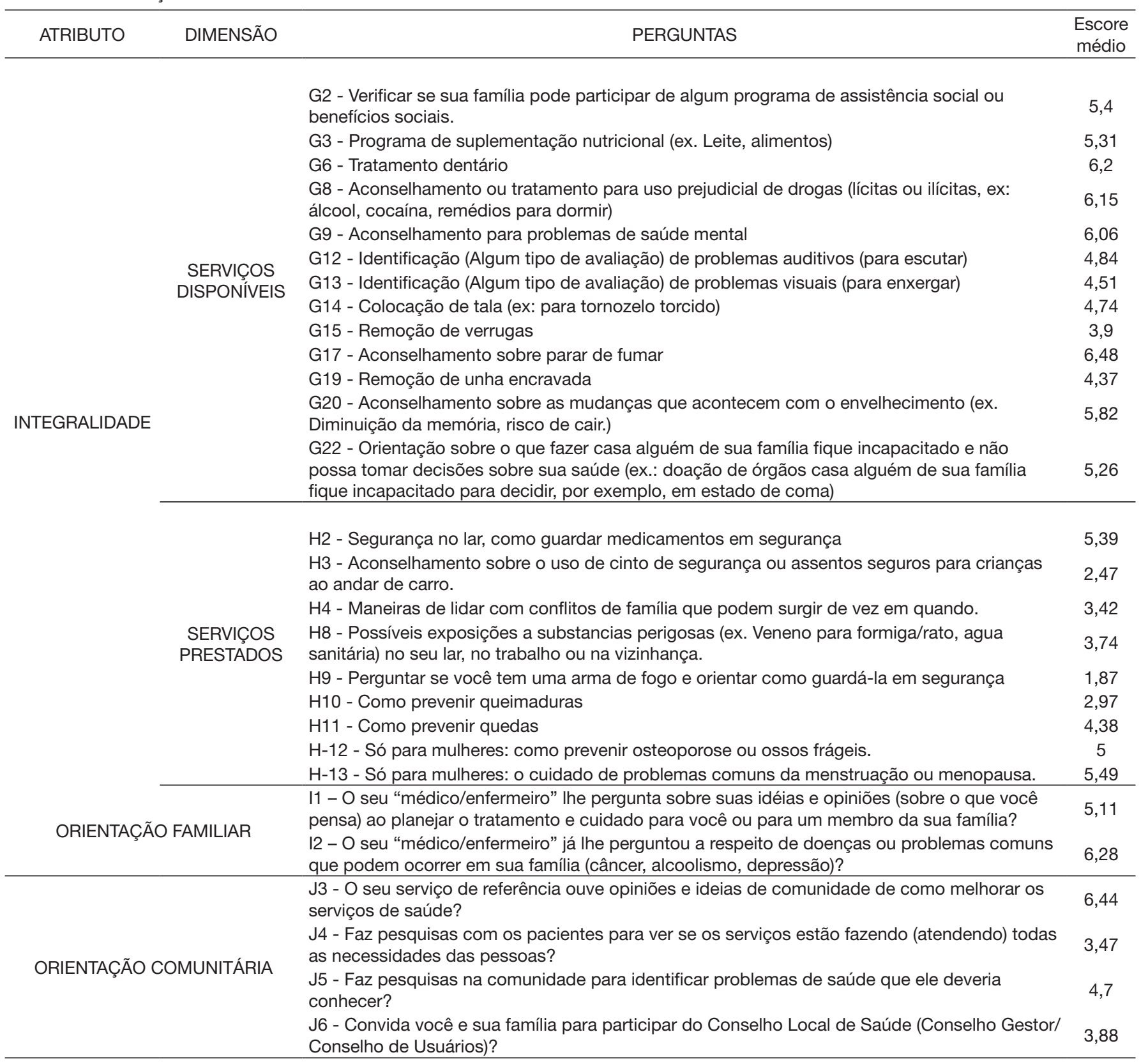

Legenda: PCAToo I = Primary Care Assesment Tool

compõe a dimensão Orientação comunitária, em que os usuários se mostraram insatisfeitos no tocante à realização de pesquisas junto a eles e suas comunidades, em relação a seus problemas de saúde, bem como no que se refere ao estímulo à participação em conselhos gestores das unidades. Outros itens importantes, que foram mal avaliados por esses usuários em outras dimensões, foram: Acessibilidade (rapidez para passar a criança em consulta e disponibilidade de aconselhamento por telefone) e Integralidade - serviços prestados (existência de programa de suplementação nutricional; identificação de deficiências visuais).

No que se refere ao acompanhamento por profissionais da APS, exceto médicos e enfermeiros, em relação à sua condição de saúde (Tabela 6), alguns itens avaliados estavam abaixo do critério de corte.

Em relação a usuários adultos e idosos, houve maior reconhecimento por parte da equipe de problemas que afetam a aparência do usuário, como falta de alguma parte do corpo, dificuldade de locomoção e problemas para movimentar alguma parte do corpo, do que para reconhecer problemas psiquiátricos, relacionados à visão, audição ou fala. Além disso, observaram-se dificuldades no reconhecimento pela equipe da necessidade de orientação de cuidadores ou do próprio usuário, que necessita de auxílio na realização de atividades cotidianas. Para responsáveis por usuários menores de 18 anos, o reconhecimento pela equipe dos problemas é maior quando relacionado à locomoção, fala, 
Tabela 5. Descrição dos itens que foram avaliados negativamente (abaixo do critério de corte) e que são prioritários para ações de melhoria para menores de 18 anos

\begin{tabular}{|c|c|c|c|}
\hline ATRIBUTO & DIMENSÃO & PERGUNTAS & $\begin{array}{l}\text { Escore } \\
\text { médio }\end{array}$ \\
\hline \multirow{3}{*}{$\begin{array}{l}\text { ACESSO DE } \\
\text { PRIMEIRO } \\
\text { CONTATO }\end{array}$} & \multirow{3}{*}{ ACESSIBILIDADE } & $\begin{array}{l}\text { C2 - Você tem que esperar muito tempo ou falar com muitas pessoas para marcar hora no(a) } \\
\text { "nome do serviço de saúde/ou nome médico/enfermeiro"? }\end{array}$ & 6 \\
\hline & & $\begin{array}{l}\text { tem que esperar mais de } 30 \text { minutos para que sua criança consulte com o médico/enfermeiro } \\
\text { (sem contar triagem ou acolhimento)? }\end{array}$ & 4,22 \\
\hline & & $\begin{array}{l}\text { C6 - Quando o "nome do serviço de saúde / ou nome médico/enfermeiro" está aberto, você } \\
\text { consegue aconselhamento rápido pelo telefone se precisar? }\end{array}$ & 4,22 \\
\hline \multirow{3}{*}{ COORDENAÇÃO } & \multirow{3}{*}{$\begin{array}{l}\text { INTEGRALIDADE } \\
\text { DO CUIDADO }\end{array}$} & E4 - O "médico/enfermeiro" de sua criança ficou sabendo quais foram os resultados desta consulta? & 5,24 \\
\hline & & $\begin{array}{l}\text { E5 - Depois desta consulta com o especialista ou serviço especializado, o seu "médico/ } \\
\text { enfermeiro" conversou com você sobre o que aconteceu durante esta consulta? }\end{array}$ & 3,33 \\
\hline & & $\begin{array}{l}\text { E6 - O seu "médico/enfermeiro" pareceu interessado na qualidade do cuidado que foi dado a } \\
\text { sua criança no especialista ou serviço especializado? }\end{array}$ & 5,24 \\
\hline \multirow{2}{*}{ INTEGRALIDADE } & \multirow[t]{2}{*}{$\begin{array}{l}\text { SERVIÇOS } \\
\text { PRESTADOS }\end{array}$} & $\begin{array}{l}\text { H3 - Mudanças do crescimento e desenvolvimento da criança, isto é, que coisas você deve } \\
\text { esperar de cada idade. Por exemplo, quando a criança vai caminhar, controlar o xixi ... }\end{array}$ & 6,43 \\
\hline & & $\begin{array}{l}\text { H5- Maneiras para manter sua criança segura, como: Evitar tombos de altura ou manter as } \\
\text { crianças afastadas do fogão }\end{array}$ & 5 \\
\hline \multicolumn{2}{|c|}{ ORIENTAÇÃO FAMILIAR } & $\begin{array}{l}\text { I2 - O seu "médico/enfermeiro" já Ihe perguntou sobre doenças ou problemas que existam na } \\
\text { família de sua criança (câncer, alcoolismo, depressão)? }\end{array}$ & 6,19 \\
\hline \multirow{2}{*}{\multicolumn{2}{|c|}{ ORIENTAÇÃO COMUNITÁRIA }} & $\begin{array}{l}\text { J3 - Faz pesquisas na comunidade para identificar problemas de saúde que ele deveria } \\
\text { conhecer? }\end{array}$ & 4,52 \\
\hline & & $\begin{array}{l}\text { J4 - Convida membros da família a participar do Conselho Local de Saúde (Conselho Gestor/ Conselho } \\
\text { de Usuários)? }\end{array}$ & 2,86 \\
\hline
\end{tabular}

Legenda: PCATool = Primary Care Assesment Tool

Tabela 6. Média dos escores, por faixa etária, quanto ao acompanhamento de suas deficiências e/ou incapacidade por profissionais da APS, exceto médicos e enfermeiros

\begin{tabular}{|c|c|c|c|}
\hline ATRIBUTO & PERGUNTAS & $\begin{array}{l}\text { Usuários } \\
\leq 18 \text { anos }\end{array}$ & $\begin{array}{l}\text { Usuários } \\
>18 \text { anos }\end{array}$ \\
\hline Grau de Afiliação & $\begin{array}{l}\text { Há algum profissional de saúde, que NÃO seja médico ou enfermeiro, no serviço de saúde em que } \\
\text { você é atendido que também é responsável por seu atendimento de saúde? }\end{array}$ & 2,63 & 2,10 \\
\hline \multirow{3}{*}{$\begin{array}{c}\text { Acesso de } \\
\text { Primeiro Contato } \\
\text { - Utilização }\end{array}$} & $\begin{array}{l}\text { Quando você necessita do profissional que melhor conhece a sua história, o médico ou } \\
\text { enfermeiro tem que encaminhar você obrigatoriamente? }\end{array}$ & 3,7 & 5,25 \\
\hline & $\begin{array}{l}\text { É fácil marcar hora para uma avaliação com o profissional que melhor conhece a sua história } \\
\text { neste serviço que você utiliza? }\end{array}$ & 8,15 & 5,73 \\
\hline & $\begin{array}{l}\text { É difícil para você conseguir atendimento rapidamente com o profissional que melhor conhece a } \\
\text { sua história deste serviço quando pensa que é necessário? }\end{array}$ & 2,59 & 6,15 \\
\hline \multirow[t]{2}{*}{ Longitudinalidade } & $\begin{array}{l}\text { Quando você vai neste serviço em que é atendido pelo(a) profissional que melhor conhece a sua } \\
\text { história, é sempre este profissional que o atende todas as vezes? }\end{array}$ & 9,26 & 6,46 \\
\hline & O profissional que melhor conhece a sua história sabe quais problemas são mais importantes para você? & 9,26 & 6,98 \\
\hline \multirow{11}{*}{$\begin{array}{l}\text { Integralidade } \\
\text { - Serviços } \\
\text { Disponíveis }\end{array}$} & Caso tenha problemas de locomoção, alguém deste serviço reconhece esta necessidade? & 10 & 8 \\
\hline & $\begin{array}{l}\text { Caso tenha problemas de uso das mãos ou braços para realizar tarefas do dia a dia, alguém deste } \\
\text { serviço reconhece esta necessidade? }\end{array}$ & - & 5,88 \\
\hline & Caso tenha problemas para falar, alguém deste serviço reconhece esta necessidade? & 10 & 5 \\
\hline & Caso tenha problemas psiquiátricos, alguém deste serviço reconhece esta necessidade? & 9,17 & 4,58 \\
\hline & Caso tenha problemas intelectuais, alguém deste serviço reconhece esta necessidade? & 6,67 & 6,67 \\
\hline & Caso tenha problemas para ouvir, alguém deste serviço reconhece esta necessidade? & _- & 3,33 \\
\hline & Caso tenha problemas para enxergar, alguém deste serviço reconhece esta necessidade? & 10 & 5,25 \\
\hline & $\begin{array}{l}\text { Caso tenha problemas relacionados à falta de alguma parte do seu corpo, alguém deste serviço } \\
\text { reconhece esta necessidade? }\end{array}$ & - & 9,05 \\
\hline & $\begin{array}{l}\text { Caso tenha problemas para movimentar alguma parte do corpo, alguém deste serviço reconhece } \\
\text { esta necessidade? }\end{array}$ & 10 & 8,13 \\
\hline & Caso necessite, a pessoa que o auxilia recebe orientação do serviço de saúde para prestar cuidados? & 6,67 & 4,44 \\
\hline & Caso ajude, você recebe orientação do serviço de saúde para prestar estes cuidados? & 5 & 3,86 \\
\hline
\end{tabular}

Legenda: APS = Atenção Primária em Saúde; Nota: Alguns itens não puderam ser calculados para usuários abaixo de 18 anos, em virtude do pequeno número de usuários participantes nessa faixa etária e da impossibilidade de calcular respostas de entrevistados quando esses respondem a menos de $50 \%$ dos itens 
visão, movimentação de alguma parte do corpo e a problemas de ordem psiquiátrica, e menor, porém na média, para problemas intelectuais.

\section{DISCUSSÃO}

No que se refere ao perfil sociodemográfico dos usuários, vale destacar que, no Brasil, há maior presença de mulheres que buscam, com maior frequência que os homens, os serviços de atenção à saúde e que deixam de realizar as atividades habituais por motivo de saúde ${ }^{(21)}$. Ao mesmo tempo, verificou-se que a maior participação de mulheres ${ }^{(11,16,17)}$ e de adultos e idosos ${ }^{(9)}$ nesta pesquisa é semelhante a outros estudos. Outra característica dos participantes é que se declararam pertencentes à classe " $\mathrm{C}$ ", conforme perfil de renda da população das áreas periféricas na região de saúde estudada ${ }^{(22)}$. A baixa renda, também apontada por participantes em outros estudos ${ }^{(11,23)}$, aliada à baixa inserção no mercado de trabalho e à pouca qualificação profissional dos participantes com deficiência, pode sugerir vulnerabilidade social dos entrevistados. Em consonância, estudos apontam que as pessoas com deficiência $(\mathrm{PcD})$ estão pouco representadas no mercado de trabalho, ocupam posições subalternas e recebem menores salários que outros trabalhadores ${ }^{(24-26)}$. Destacou-se, ainda, no presente estudo, o baixo acesso dos usuários com deficiência ao benefício de prestação continuada, o que pode decorrer da falta de informação sobre os critérios para requisição e concessão do benefício.

Observou-se que a participação dos usuários em grupos comunitários é pequena, sendo mais frequentemente apontada nas unidades de saúde às quais os entrevistados estão vinculados, o que pode sugerir a escassez de parcerias entre serviços e setores da sociedade para fomentar a participação social das $\mathrm{PcD}$, conforme relatado em outro estudo ${ }^{(18)}$.

Quanto à percepção sobre os diferentes atributos da APS, não se verificou diferença estatisticamente significante quando comparados usuários com e sem deficiência. A ausência de diferenças também foi verificada em estudo que avaliou a APS sob a ótica dos responsáveis por crianças e adolescentes com e sem deficiência física ${ }^{(18)}$.

O Grau de afiliação foi bem avaliado pelos usuários em geral (com e sem deficiência), quando se trata de atenção prestada por médicos e enfermeiros. De modo geral, os usuários pareceram reconhecer os serviços ou os profissionais de saúde como referência no cuidado ${ }^{(11,18)}$.

Do mesmo modo, o atributo Acesso de primeiro contato: utilização foi bem avaliado pelos entrevistados, o que corrobora os resultados encontrados em outros estudos ${ }^{(16,17,27)}$.

$\mathrm{O}$ atributo Acesso primeiro contato: acessibilidade, que baliza a disponibilidade do serviço para o usuário, bem como o acolhimento e a humanização, foi um dos atributos pior avaliado, assim como ocorreu em outras pesquisas ${ }^{(9,16,17)}$. Esses resultados decorrem em especial das pontuações muito baixas em itens como "não abrir aos sábados e domingos", "em dias úteis após as 20 h", "não fornecer possibilidade de contato telefônico nesses períodos" ${ }^{\prime(16,28-30)}$, que expressam questões estruturais que levam os usuários a ter pouca acessibilidade aos serviços. No que se refere às pessoas com deficiência, este atributo teve um escore ainda mais baixo, quando comparado ao daquelas sem deficiência, embora sem diferença estatística significante. Estes dados sugerem que no caso das $\mathrm{PcD}$ ficam ainda mais nítidas as dificuldades enfrentadas no acesso aos serviços de saúde, enfatizando as iniquidades às quais estes indivíduos estão sujeitos ${ }^{(23)}$.

À semelhança do que ocorreu em outros trabalhos, o atributo Longitudinalidade foi avaliado de modo satisfatório, por usuários em geral ${ }^{(16)}$, por menores de 18 anos $^{(27)}$ e por idosos $^{(13)}$. A boa avaliação desse atributo é fundamental para o adequado tratamento, limitando encaminhamentos desnecessários para serviços de maior complexidade ${ }^{(24)}$, o que sugere o uso da APS como fonte habitual para atender a todos os problemas de saúde, acolhendo o usuário de forma holística por anos ${ }^{(15)}$.

A Coordenação da atenção: integralidade de cuidado tem recebido distintas avaliações considerando os diversos estudos. No presente trabalho, esse atributo foi avaliado acima do critério de corte por usuários em geral e por adultos e idosos, enquanto que relativamente adequado em estudo pregresso ${ }^{(27)}$ e mal avaliado em estudo anterior ${ }^{(17)}$. $\mathrm{O}$ atributo Coordenação da atenção: sistema de informação também teve seu escore acima do critério de corte no atual estudo, quando avaliado por adultos, por idosos e de forma geral, semelhante ao verificado em outro estudo ${ }^{(2)}$. A coordenação deve se dar ao longo do percurso terapêutico e assegurar a continuidade da atenção em diferentes pontos, o que indica a utilização da informação e de serviços adequados pelos usuários ${ }^{(17)}$.

No presente estudo, o atributo Integralidade: serviços disponíveis foi avaliado abaixo do valor de corte. De modo similar, em outras pesquisas foram considerados também como pouco disponíveis, serviços como remoção de verruga ${ }^{(13,16)}$, aconselhamento/tratamento quanto ao uso prejudicial de drogas, colocação de talas, identificação de problemas auditivos e visuais, aconselhamento em relação a mudanças referentes ao processo de envelhecimento, orientação sobre cuidados a familiares com incapacidades ${ }^{(16)}$.

Salienta-se que os usuários adultos e idosos com deficiência consideraram que os serviços não reconhecem grande parte de suas demandas específicas, especialmente aquelas não manifestas em sua aparência física como problemas para ouvir, enxergar, falar, problemas psiquiátricos, para uso dos membros superiores em atividades do dia a dia ou ainda quanto à necessidade de orientação a cuidadores. $\mathrm{O}$ não reconhecimento pela equipe dessas condições pode indicar a invisibilidade para os serviços das PcD e de suas dificuldades na vida cotidiana, impossibilitando a efetivação do papel da APS como ordenadora da atenção em rede, inclusive para esse segmento populacional ${ }^{(3,4)}$.

O menor escore obtido para o atributo integralidade: serviços prestados também foi identificado em outras pesquisas e esteve relacionado à falta de aconselhamento sobre: segurança infantil no carro, ingestão de substâncias perigosas, acidentes domésticos como queimaduras ${ }^{(16,23)}$, quedas e porte de armas de fogo ${ }^{(16)}$.

No que diz respeito aos atributos derivados, a Orientação comunitária foi avaliada abaixo do escore de corte pelos usuários em geral da mesma forma que em outros estudos ${ }^{(1,9,16,18,28)}$. Os usuários, à semelhança do que foi encontrado em outras pesquisas, mostraram-se insatisfeitos com os serviços no tocante 
a pesquisas junto a eles e suas comunidades em relação a seus problemas de saúde ${ }^{(1,28)}$, ao desempenho dos serviços e ao estímulo à participação em conselhos gestores das unidades ${ }^{(28)}$.

$\mathrm{O}$ atributo Orientação familiar também foi avaliado abaixo do escore de corte pelos usuários em geral e em especial para os adultos entre 19 e 59 anos, em conformidade com resultados obtidos em outras pesquisas ${ }^{(1,9,11,16,18,28,29)}$. Isso pode sugerir a fragilidade dos serviços no que diz respeito ao trabalho a partir da realidade social e familiar dos usuários e da participação de indivíduos e suas famílias no processo de cuidado, indicando a necessidade de aprimoramento da atenção orientada a partir dos pressupostos da estratégia de saúde da família.

Salienta-se que o escore geral, embora avaliado abaixo da média, não se distanciou sensivelmente do valor de referência, devido à boa avaliação dos serviços, em especial pelos responsáveis por menores de 18 anos, enquanto que a avaliação próxima (porém acima) do valor de corte para o escore essencial recebeu avaliação positiva dos serviços por maiores de 60 anos e por menores de 18 anos. Isso sugere a necessidade de um estudo mais aprofundado acerca das atividades e ações realizadas pelos serviços e o quanto essas contemplam necessidades e demanda da diversidade de seus usuários.

Sendo assim, assinala-se que os atributos e dimensões mais bem avaliados foram grau de afiliação, acesso primeiro contato: utilização e longitudinalidade, seguidos de coordenação da atenção: sistema de informação. Além disso, não foram verificadas diferenças significantes no que se refere à percepção dos usuários com e sem deficiência.

Estes dados sugerem que a atenção desenvolvida nos serviços tem atendido parcialmente às expectativas e necessidades de cuidado, indicando, por um lado, que os serviços são referência para esses usuários e respondem suas demandas e necessidades de atenção. Por outro lado, percebe-se que tanto o escore geral quanto o essencial refletem que existe uma grande margem para melhoria da atenção prestada pelos serviços pesquisados, já que a nota máxima possível não foi alcançada, ou seja, existem atributos que podem ser aprimorados.

Neste sentido, destaca-se como a dimensão mais mal avaliada pelos usuários acesso primeiro contato: acessibilidade, principalmente no que diz respeito a questões estruturais, indicando necessidade de se repensar, do ponto de vista da gestão dos serviços, estratégias a serem adotadas para garantir o acesso pleno de todos os usuários à APS e ao sistema de saúde, bem como a atenção a diferentes demandas.

Merece destaque também o fato de os serviços terem dificuldades para reconhecer questões específicas relacionadas às deficiências, principalmente para adultos e idosos, parte significativa da população que, ao longo da vida, poderá enfrentar problemas relacionados à diminuição de sua capacidade funcional com repercussões para as suas condições de saúde e de autonomia para o cuidado de si ou de outros.

Dentre as limitações do estudo, destacam-se os distintos graus de disponibilidade dos serviços para acolher pesquisadores e alunos, a extensão do questionário e o tempo requerido para sua aplicação, o que dificultou a participação de um número maior de indivíduos na pesquisa e, consequentemente, o aprofundamento de fatores relacionados às percepções de usuários sobre a atenção recebida.

\section{CONCLUSÃO}

A percepção dos usuários, em especial das pessoas com deficiência na avaliação dos atributos essenciais e derivados da atenção básica, indica que os menores de 18 anos apresentaram o maior número de atributos e dimensões bem avaliadas, seguidos por idosos. Isso corresponde às faixas etárias que mais necessitam de atenção e cuidado e que contam com programas mais específicos, o que pode favorecer o acesso ao sistema e a continuidade de cuidados nesse nível assistencial. Quando comparados usuários com e sem deficiência, não foram observadas diferenças estatisticamente significantes.

\section{REFERÊNCIAS}

1. Alencar MN, Coimbra LC, Morais APP, Silva AAM, Pinheiro SRA, Queiroz RCS. Avaliação do enfoque familiar e orientação para a comunidade na Estratégia Saúde da Família. Ciênc Saúde Colet. 2014;19(2):353-64. http:// dx.doi.org/10.1590/1413-81232014192.08522012.

2. Carneiro MSM, Melo DMS, Gomes JM, Pinto FJM, Silva MGC. Avaliação do atributo coordenação da Atenção Primária à Saúde: aplicação do PCATool a profissionais e usuários. Saúde Debate. 2014;38(esp):279-95. http://dx.doi.org/10.5935/0103-1104.2014S021.

3. Brasil. Ministério da Saúde. PNAB: Política Nacional de Atenção Básica [Internet]. Brasília: Ministério da Saúde; 2012 [citado 2016 Out 13]. (Série E Legislação em Saúde). Disponível em: http://dab.saude.gov.br/ portaldab/biblioteca.php?conteudo=publicacoes/pnab

4. Brasil. Ministério da Saúde. Portaria 793, de 24 de abril de 2012. Institui a Rede de Cuidados à Saúde da Pessoa com Deficiência no âmbito doa Sistema Único de Saúde [Internet]. 2012 [citado 2016 Out 13]. Disponível em: http://bvsms.saude.gov.br/bvs/saudelegis/gm/2012/prt0793_24_04_2012. html

5. Harzheim E, Oliveira MMC, Agostinho MR, Hauser L, Stein AT, Gonçalves MR, et al. Validação do instrumento de avaliação da atenção primária à saúde: PACTool-Brasil adultos. Rev Bras Med Fam Comunidade. 2013;8(29):274-84. http://dx.doi.org/10.5712/rbmfc8(29)829.

6. Starfield B, Xu J, Shi L. Validating the Adult Primary Care Assessment Tool. JFP. 2001;50(2):161-75.

7. Fracolli LA, Gomes MFP, Nabão FRZ, Santos MS, Cappellini VK, Almeida ACC. Instrumentos de avaliação da Atenção Primária à Saúde: revisão de literatura e metassíntese. Cien Saude Colet. 2014;19(12):4851-60. PMid:25388193. http://dx.doi.org/10.1590/1413-812320141912.00572014.

8. Ribeiro LCC, Rocha RL, Ramos-Jorge ML. Acolhimento às crianças na atenção primária à saúde: um estudo sobre a postura dos profissionais das equipes de saúde da família. Cad Saude Publica. 2010;26(12):2316-22. PMid:21243226. http://dx.doi.org/10.1590/S0102-311X2010001200010.

9. Sala A, Luppi CG, Simões O, Marsiglia RG. Integralidade e atenção primária à saúde: avaliação na perspectiva dos usuários de unidades de saúde do município de São Paulo. Saude Soc. 2011;20(4):948-60. http:// dx.doi.org/10.1590/S0104-12902011000400012.

10. Villa TC, Ruffino A No, Scatena LM, Andrade RL, Brunello ME, Nogueira JA, et al. Desempenho dos serviços de saúde para o tratamento da tuberculose no Brasil: um estudo transversal. BMC Health Serv Res. 2011;11(1):241. PMid:21955523. http://dx.doi.org/10.1186/1472-6963-11-241.

11. Leão CDA, Caldeira AP. Avaliação da associação entre qualificação de médicos e enfermeiros em atenção primária em saúde e qualidade da atenção. Cien Saude Colet. 2011;16(11):4415-23. PMid:22124822. http:// dx.doi.org/10.1590/S1413-81232011001200014. 
12. Oliveira EB, Bozzetti MC, Hausser L, Ducan BB, Harzheim E. Avaliação da qualidade do cuidado a idosos nos serviços da rede pública de atenção primária à saúde de Porto Alegre, Brasil. RBMFC. 2013;8(29):264-73.

13. Araújo LUA, Gama ZAS, Nascimento FLA, Oliveira HFV, Azevedo WM, Almeida HJB Jr. Avaliação da qualidade da atenção primária à saúde sob a perspectiva do idoso. Cien Saude Colet. 2014;19(8):3521-32. PMid:25119091. http://dx.doi.org/10.1590/1413-81232014198.21862013.

14. Paes NA, Silva CS, Figueiredo TMRM, Cardoso MAA, Lima JO. Satisfação dos usuários hipertensos com os serviços da rede de atenção primária no Brasil: um estudo de validação. Rev Panam Salud Publica. 2014;36(2):8793. PMid:25345529.

15. Frank BRB, Viera CS, Ross C, Obregón PL, Toso BRGO. Avalição da longitudinalidade em unidades de Atenção Primária à Saúde. Saúde Debate. 2015;39(105):400-10. http://dx.doi.org/10.1590/0103-110420151050002008.

16. Silvia AS, Francolli LA. Avaliação da estratégia de saúde da família perspectiva dos usuários em Minas Gerais, Brasil. Saúde Debate. 2014;38(103):692705. http://dx.doi.org/10.5935/0103-1104.20140064.

17. Lima EFA, Sousa AI, Primo CC, Leite FMC, Lima RCD, Maciel ELN. Avaliação dos atributos da atenção primária na perspectiva das usuárias que vivenciam o cuidado. Rev Latino-Am Enfermagem. 2015;23(3):553-9. http://dx.doi.org/10.1590/0104-1169.0496.2587.

18. Quaresma FRP, Stein AT. Attributes of primary health care provided to children/adolescents with and without disabilities. Ciênc. Saúde Coletiva. 2015;20(8):2561-8.

19. Brasil. Manual do Instrumento de Avaliação da Atenção Primária à Saúde: Primary Care Assessment Tool PCATool-Brasil. Brasília: Ministério da Saúde; 2010

20. ABEP: Associação Brasileira de Empresas de Pesquisa. Critério Brasil [Internet]. São Paulo: ABEP; 2014 [Citado em 2015 Ago 21]. Disponível em: http://www.abep.org/criterio-brasil

21. IBGE: Instituto Brasileiro de Geografia e Estatística. Pesquisa Nacional de Saúde - 2013: acesso e utilização dos serviços de saúde, acidentes e violências - Brasil, grandes regiões e unidades da federação. Rio de Janeiro: IBGE; 2015. $100 \mathrm{p}$.

22. São Paulo. Secretaria Municipal de Saúde. Coordenadoria Regional de Saúde Centro Oeste. Relatório de Gestão 2005-2012. São Paulo: 2012.
23. Araújo JP, Viera CS, Toso BRGO, Collet N, Nassar PO. Avaliação dos atributos de orientação familiar e comunitária na saúde da criança. Acta Paul Enferm. 2014;27(5):404-6.

24. Aoki M, Oliver FC, Nicolau SM. Considerações acerca das condições de vida das Pessoas com deficiência a partir de um levantamento em uma unidade básica de saúde de um bairro periférico do município de São Paulo. O Mundo da Saúde. 2011;35(2):169-178.

25. Stoneman J, Lysaght R. Supported education: a means for enhancing employability for adults with mental illness. Work. 2010;36(2):257-9. PMid:20634619.

26. Toldrá RC, Santos MC. People with disabilities in the labor market: facilitators and barriers. Work. 2013;45(4):553-63. PMid:23676332.

27. Leão CDA, Caldeira AP, Oliveira MMC. Atributos da atenção primária na assistência à saúde da criança: avaliação dos cuidadores. Rev Bras Saude Mater Infant. 2011;11(3):323-34. http://dx.doi.org/10.1590/S151938292011000300013.

28. Oliveira VBCA, Verissimo MLR. Assistência à saúde da criança segundo suas famílias: comparação entre modelos de Atenção Primária. Rev Esc Enferm USP. 2015;49(1):30-6.

29. Paula FA, Silva CCR, Santos DF, Martins OA Fo, Andrade RA. Avaliação da atenção a saúde do adulto em um município-polo do Vale do Jequitinhonha MG. Saúde Debate. 2015;39(106):802-14. http://dx.doi.org/10.1590/01031104201510600030020 .

30. Fracolli LA, Muramatsu JM, Gomes MFP, Nabão FRZ. Avaliação dos atributos da Atenção Primária à Saúde num município do interior do Estado de São Paulo - Brasil. O Mundo da Saúde. 2015;39(1):54-61.

\section{Contribuição dos autores}

MHMA e FCO participaram do desenho, concepção, análise e preparação dos originais; AS e DRMA participaram do desenho, concepção, análise e preparação dos originais; $R C T, S P, S K$ e AMO participaram da análise e preparação dos originais. 